\title{
Automatic Generation of 3D-Printed Reactionware for Chemical Synthesis Digitization using ChemSCAD
}

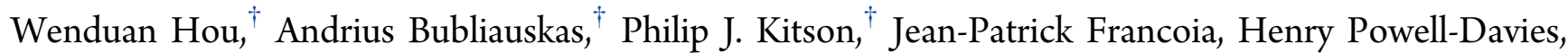
Juan Manuel Parrilla Gutierrez, Przemyslaw Frei, J. Sebastián Manzano, and Leroy Cronin*

Cite This: ACS Cent. Sci. 2021, 7, 212-218

Read Online

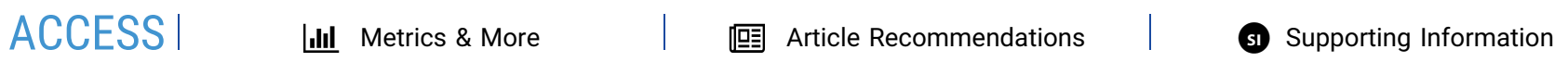

ABSTRACT: We describe a system, ChemSCAD, for the creation of digital reactors based on the chemical operations, physical parameters, and synthetic sequence to produce a given target compound, to show that the system can translate the gram-scale batch synthesis of the antiviral compound Ribavirin (yield 43\% over three steps), the narcolepsy drug Modafinil (yield 60\% over three steps), and both batch and flow instances of the synthesis of the anticancer agent Lomustine (batch yield $65 \%$ over two steps) in purities greater than or equal to $96 \%$. The syntheses of compounds developed using the ChemSCAD system, including reactor designs and analytical data, can be stored in a database repository, with the information necessary to critically evaluate and improve upon reactionware syntheses being easily shared and versioned.

\section{INTRODUCTION}

Digital chemistry aims to define a hard link from the top abstraction layer in chemistry down to the synthesis, but this is difficult in traditional glassware, since it is not possible to explicitly link the architecture with the unit operations. By a three-dimensional printing (3DP) of the synthesis modules in the precise order to affect the synthesis, it is possible to create digitally encoded reactors for chemical synthesis in "reactionware". However, the creation of these devices requires a specific skillset for computer-aided design (CAD) modeling, which few synthetic chemists have. The past decade has seen the range and scope of the exploitation of 3DP, also known as additive manufacturing, techniques expand vastly, ${ }^{1}$ moving beyond the preserve of industrial manufacturing or prototyping applications, to play a significant role in many areas of science and engineering. ${ }^{2}$ Driving this expansion has been the adoption of an "open source" approach to 3DP development, whereby an enthusiastic base of practitioners with access to relatively cheap 3D printed equipment has been able to openly share their expertise and adaptations of methods to produce new materials and functional devices. The applications of 3DP have become increasingly prevalent within research laboratories to produce highly specialized electronic, ${ }^{3}$ microfluidic, ${ }^{4}$ and pneumatic devices. ${ }^{5,6}$ This expansion has so far, however, only had a limited impact in the area of synthetic chemistry.

In 2012, our group pioneered the concept of laboratory-scale $3 \mathrm{D}$ printed chemical reactors, known as reactionware, where the unique properties of $3 \mathrm{DP}$ as a manufacturing technique allow the researcher to control and rapidly iterate the geometry, topology, and composition of the manufactured reactors to direct the synthetic outcome of reactions. ${ }^{7}$ Using this approach we were able to show that 3DP reactors can be employed for a wide range of synthetic applications from flow synthesis ${ }^{8}$ and standard batch reactions ${ }^{9}$ to opening up new synthetic strategies in high-throughput hydrothermal synthesis. ${ }^{10,11}$ Recently, we developed a standardized approach to the design and implementation of $3 \mathrm{D}$ printed reactors to achieve complete multistep organic and inorganic syntheses via a cartridge-based approach. ${ }^{12}$ This led to the creation of monolithic "chemical generators", which allowed chemical products to be obtained on-demand with minimal human intervention to achieve the desired synthesis. ${ }^{13}$ This approach was used to demonstrate reactionware's applicability for the production of useful and high-value materials in good yields and purities. Reactionware bypasses traditional sophisticated manufacturing centers while also facilitating the translation of traditional glassware synthesis into a platform-independent digital code, creating digital blueprints that can be part of an online library of verified, synthesisspecific reactor designs available globally.

\section{RESULTS AND DISCUSSION}

One major barrier to the further adoption of 3DP as a tool in the synthetic chemistry laboratory is the design of the reactors necessary for any reaction sequence from scratch for each individual synthesis. Until now this process has been achieved using commercially available 3D CAD software such as AutoCAD, SolidWorks, OnShape, etc., which are intended to be versatile tools for designing complex objects, rather than specifically for reactionware devices. This means that the synthetic chemist is required to be familiar with one of these software suites in order to produce the digital designs of a monolithic reactor for their specific target compound. To address this issue, we developed

Published: January 11, 2021

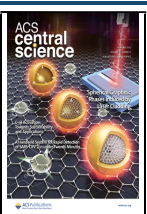




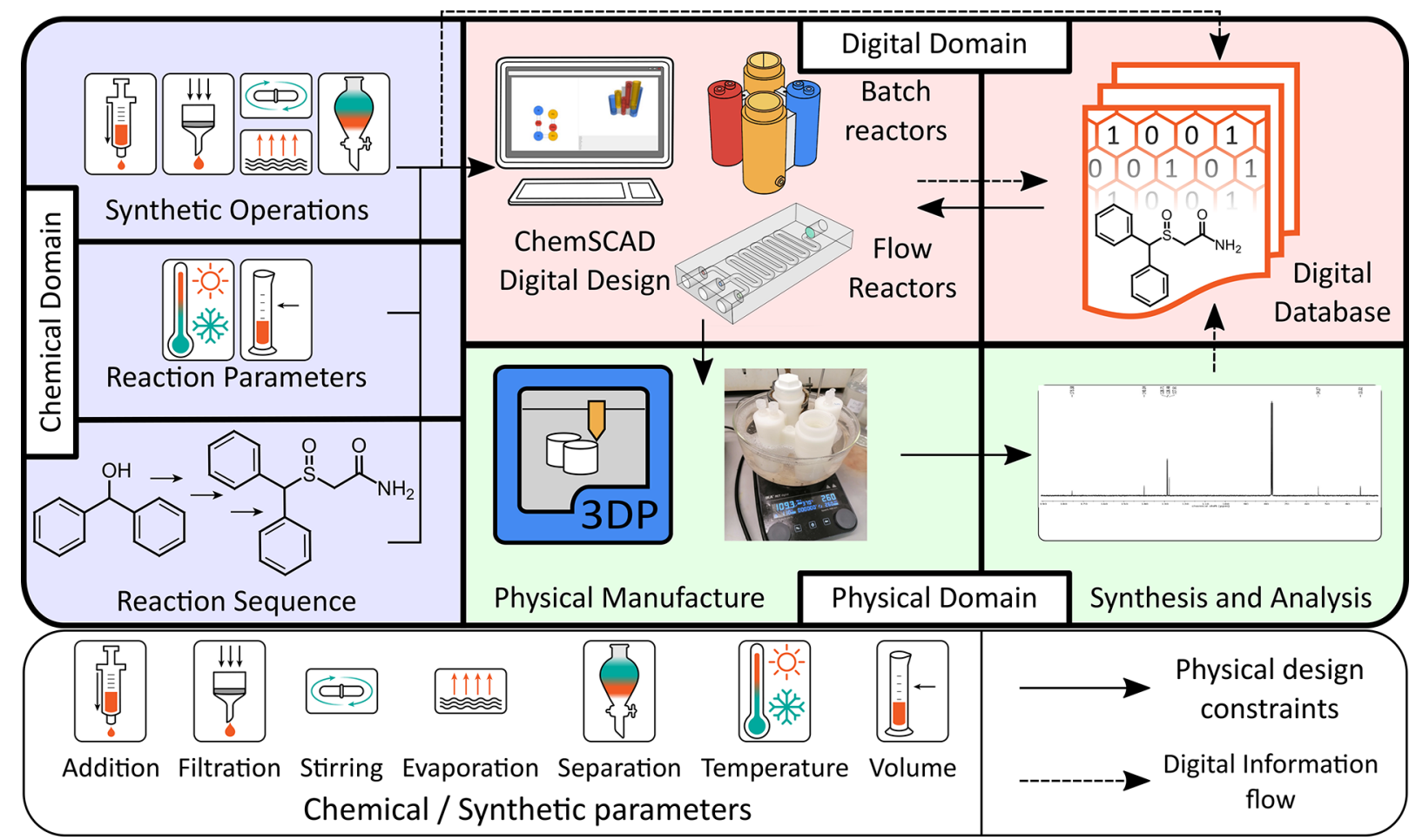

Figure 1. Schematic representation of the centrality of the ChemSCAD software to linking the chemical design of synthesis routes (Chemical Domain) to the physical manufacture of reactionware cartridges and execution of the designed syntheses (Physical Domain) via a complete digital record of the design process and associated analytical data (Digital Domain).

a system that exploits the standardized approach we have previously

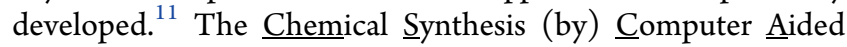
Design (ChemSCAD) system is realized through a Pythonbased software application that allows the chemist to produce multistep synthesis cartridges using only parameters relevant to the synthetic procedure. This software implementation is designed to function intuitively, requiring minimal training or CAD modeling expertise. We use this software to design reactionware cartridges for the synthesis of three pharmaceutically relevant compounds.

The software, ChemSCAD, was built using the Python programming language and is designed to run on multiple platforms (Windows, MacOS, Linux, and both 32- and 64-bit systems). ChemSCAD is a pip-installable Python software package comprised of three main contextual layers: (i) the Graphical User Interface (GUI) framework, which the user interacts with, (ii) the ccad API, and (iii) the OpenSCAD library. The API, ccad, is a bespoke Python library that generates OpenSCAD (a script-based system for producing solid $3 \mathrm{D}$ CAD objects) code that reflects the attributes of each reactor as determined by the user. ChemSCAD is a GUI wrapper around ccad to facilitate its use in a straightforward and chemically intuitive manner. Importantly, ChemSCAD also displays the STL files once OpenSCAD has rendered them (the rendering is asynchronous), displaying this in the right-hand side window. Also, since ccad is an importable Python library, it may be used without the ChemSCAD GUI wrapper for those familiar with OpenSCAD code syntax to generate more complex devices than those offered by ChemSCAD currently. Full details of the ChemSCAD system can be found in the associated Supporting Information.

ChemSCAD is built to be intuitive to the chemist, requiring no "modeling" of the final object but, rather, a focus on the chemical processes and steps required to achieve the desired synthesis, that is, the "Chemical Domain" of factors directly influencing the production of any chemical product (see Figure 1). Each of these syntheses is thus captured in the digitally defined structure of the reactor system, alongside the operational instructions defined by the geometry of the reactors. This leads to the creation of a digital repository, where all the necessary information relating to the practical execution of the desired syntheses can be gathered together (the "Digital Domain", Figure 1). This repository will act as a database of cartridge syntheses, also containing analytical data from the physical execution of the designed synthesis in the manufactured cartridge ("Physical Domain"), which can validate the procedures as designed and carried out along with a history of the development of the monolithic reactor cartridge (see the Supporting Information, Section 2.1.4). This will provide researchers with all the information necessary to critically evaluate the suitability of the reactor system. Additionally, this gives a starting point for making personalized modifications in the cartridge synthesis of any given target. By providing software where the reactor designs can be modified based solely on the physical and chemical processes, we remove the barrier of entry for the creation and development of these self-contained systems.

In our previous work ${ }^{14,15}$ we outlined a general process for the translation of individual batch reaction sequences into a monolithic cartridge, where the individual synthetic processes are telescoped and grouped into as few unit operations as possible. ChemSCAD is designed around this standard modular approach, where each element of the final reactor monolith design is built up from a library of smaller, predesigned, reactor "modules" that can carry out specific synthetic or physical processes such as filtrations, separations, etc. Individual modules can be used to achieve either specific steps in the synthesis or combinations of steps depending on the process requirements of the synthesis. Most importantly, the focus on these modules in 

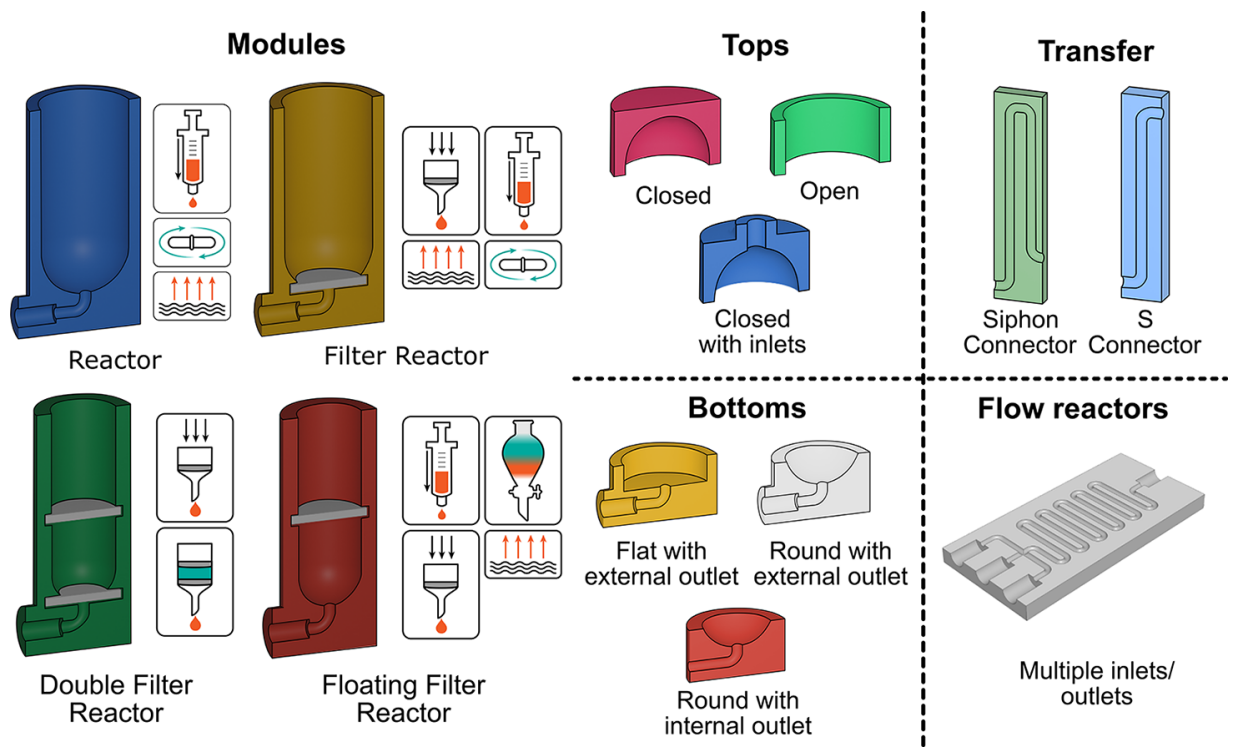

Figure 2. Structural elements used by the ChemSCAD software to build up multi-step synthesis cartridges. (left) The four basic reactor module designs with the chemical parameters that the specific module can perform. (right) Examples of variations available in the ChemSCAD software for the top, bottom, and transfer connectors of the reactor modules and a flow reactor generated using the ChemSCAD software.

ChemSCAD means the user is not required to consider the design parameters of any of building blocks but only the chemical processes required to affect the desired synthesis.

Reactionware cartridges consist of five basic types of module, four batch reaction modules, and one stand-alone flow reactor, which can be created directly in ChemSCAD. The choice of which of these modules is necessary for a given process is dictated by the operations to be carried out in the module (see Figure 2). The four basic module types and their associated functions are as follows: (i) a standard "Reactor" module consists of a simple chamber in which reactions or mixing operations can take place; (ii) a "Filter Reactor" similar to the standard Reactor but equipped with an outlet at the bottom of the chamber below a filter that can be used for separating solid and liquid phases; (iii) a "Floating Filter Reactor" that allows the position of the filter in the module chamber to be set by the user. This can be used for either solid-liquid separations or liquid-liquid separations if a hydrophobic or other selectively permeable membrane is placed in this space; (iv) a "Double Filter Reactor", which combines the features of a Filter Reactor and a Floating Filter Reactor, having a filter placed at the bottom of the chamber and a second filter dividing the main body of the module into an upper and lower chamber. This type of reactor can be used to encase a particular substance such as a drying agent or a solid supported catalyst material between the two filters to fix it in a certain location. The topology of each of these modules can be customized by the adjustment of several parameters such as the volume of the chambers, the required number of input and output ports for material transfer, the filter diameter, and the filter thickness depending on the particular components available for the fabrication of the reactionware modules. Also, on creation, the modules can be customized with several options of top and bottom types (such as open or closed tops, rounded or flat bottoms, etc.; see Figure 2).

Once the reactor chamber(s) have been created, their inputs/outputs can be connected together by a number of different types of connector, the "S-Connector", the "Siphon Connector", and the "Tube Connector". The S-Connector option creates a tube connecting an output in one module with an input in a neighboring module, where the input and output are at differing heights. This is the simplest and most common type of connection between modules. The siphon connector feature connects outputs with inputs, but in between the siphon tube rises to the height of a specified chamber to ensure no back transfer of material through the cartridge upon addition of more material to the latter chamber. Finally, the tube connector simply connects two inlets/outlets in adjacent modules adjusting the relative heights of the modules to allow the connection.

When the synthetic protocol to be translated from glassware into reactionware is considered, the paramount considerations that will determine the final global topology of the model of reactionware to be produced are the scale of the reaction, ${ }^{16}$ the physical state of the chemicals that are required to be added at each stage, the nature of the reaction mixtures present in the cartridge at each stage, and the physical or chemical processes to be performed. Designing the devices is made easy by the intuitive GUI of the ChemSCAD software, which allows the user to see both a two-dimensional representation of the connectivity and function of each of the added modules as well as a $3 \mathrm{D}$ rendering of the final module simultaneously. Also, the software allows the user to toggle easily between a linear representation of the reactor cartridge being designed and a space-saving design more optimized for the $3 \mathrm{D}$ printing manufacture of the resulting cartridge.

The scale of the reaction will determine the size of modules (i.e., smaller volumes will produce smaller modules while maintaining the required sizes for connectors/filters, etc.). Additionally, the size of the cartridges is designed based on traditional glassware synthesis, where the total volume of the reaction mixture solvent does not exceed two-thirds of the vessel in which it is contained. The manner of addition of chemical materials throughout the synthesis also plays an important role in the design. The state of reactants (i.e., solid or liquid) determines what type of inputs will be used. For example, if the reactants to be added are in solution, they can be introduced via a $5 \mathrm{~mm}$ diameter Luer adapter port using a 
a
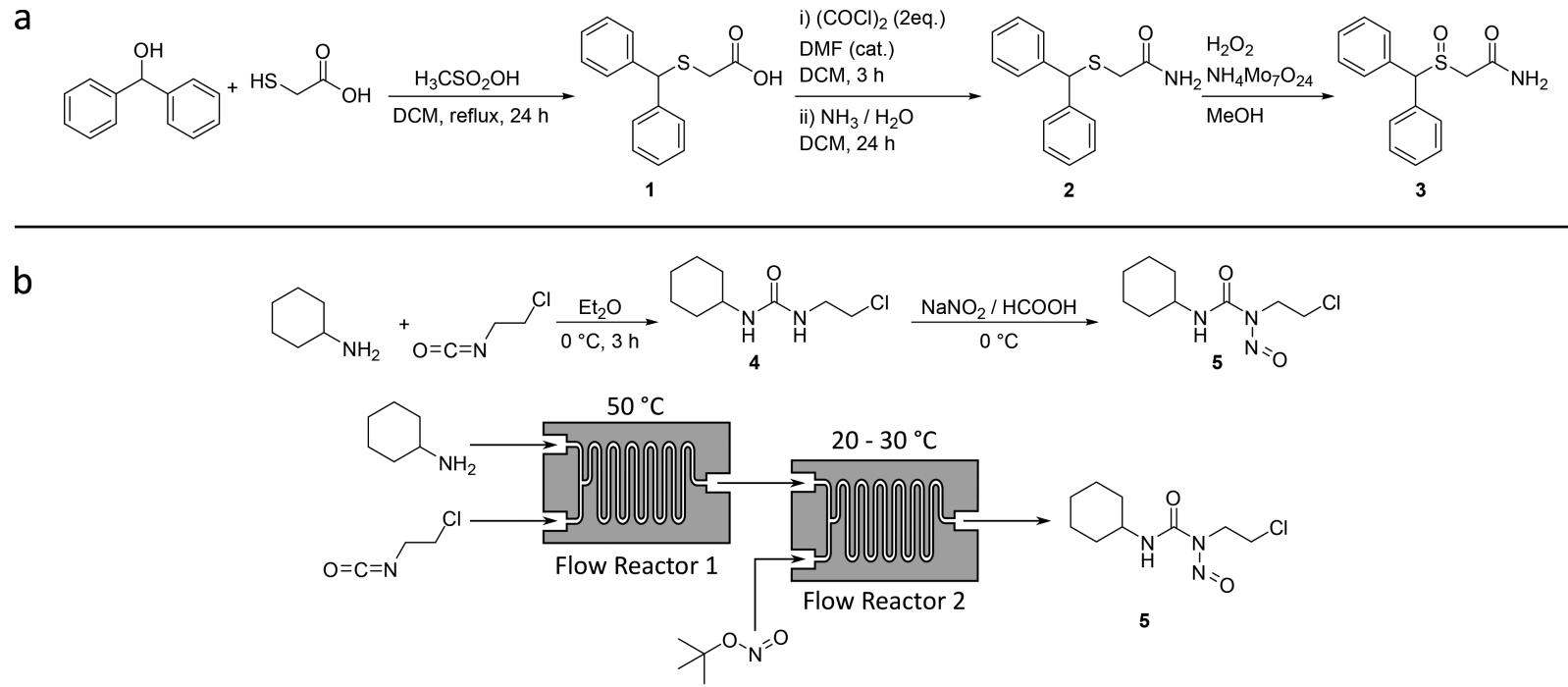

C

Figure 3. Synthetic schemes of the three compounds for which reactionware synthesis cartridges have been designed in the current study. Optimized synthetic routes to Modafinil (a), Lomustine (b), and Ribavirin (c).

syringe. This implies that an extra port must be incorporated into the design to avoid pressure buildup during liquid additions. If solid reagents are to be added, an open or threaded top can be used to allow the material to be added manually. Depending upon the synthesis steps required a threaded-cap top might be preferred for a pressurized module (e.g., filtration, evaporation).

When reactionware cartridges are designed, the nature of any liquid media to be added, whether they are neat, in solution, or in suspension, must be carefully considered, as this can affect material transfer from one module to another. In ChemSCAD the diameter of the connector channels can be manually adjusted from default values if necessary. To optimize separation processes, the differing physical dimensions of nonprinted parts such as glass filters, hydrophobic frits, etc. need to be taken into account when Filter Reactors are designed. All of these parameters can be easily adjusted in ChemSCAD. The type of chemical processes will determine the class of the module. For example, simple mixing will preferably use a Reactor module instead of a filter module. Product isolation/purification by crystallization as well as vacuum distillation is feasible in both Reactor and Filter Reactor modules. Moreover, heating, cooling, and mixing can be carried out in all module types.

To demonstrate the function of the ChemSCAD process, we designed monolithic reactionware cartridges for the batch synthesis of Ribavirin, an antiviral medication used to treat respiratory syncytial virus (RSV) infection, hepatitis C, and some viral hemorrhagic fevers; ${ }^{17}$ Modafinil, a narcolepsy drug; ${ }^{18}$ and Lomustine, an alkylating nitrosourea compound widely used in chemotherapy. ${ }^{19}$ Lomustine was also synthesized in flow. Furthermore, we demonstrate the effective synthesis of these compounds in the designed cartridges as shown in Figure 3.

The three syntheses were chosen to contain robust reactions that give high overall yields in glassware through batch operations compatible with reactionware modules. The synthetic routes were first validated by traditional glassware procedures, where the necessary unit operations ${ }^{20}$ and process parameters ${ }^{21}$ (such as solvent volumes, filtrations, and evaporations) were identified. These operations defined the type and sequence of modules produced using only the predetermined structural elements available in ChemSCAD.

Modafinil is widely used in the treatment of narcolepsy and other sleep-related disorders. ${ }^{22}$ There are many synthetic routes toward Modafinil, a lot of which are difficult for a synthetic chemist. Some of the synthetic pathways require the use of bench-unstable reactants or difficult-to-perform productisolation techniques. ${ }^{23,24}$ Here we present a new synthetic route that can be easily performed in a $3 \mathrm{D}$ printed reactionware monolith. The pathway starts with cheap starting materials and throughout the synthesis uses easy-to-handle synthetic reagents and techniques. The pathway avoids biphasic separations and is composed of simple heating, stirring, cooling, reduced pressure solvent evaporation, precipitation, and crystallization steps. In the first step in the synthesis of Modafinil the starting materials thioglycolic acid and diphenylmethanol are combined in dichloromethane (DCM) and heated under reflux to yield the first intermediate (benzhydrylthio)acetic acid (compound 1). ${ }^{25}$ The resulting reaction mixture is then concentrated under reduced pressure and dissolved in a basic aqueous media. All of these processes can be achieved in a single Reactor module, fitted with a $3 \mathrm{D}$ printed polypropylene high surface condenser for the initial reflux. The first module, therefore, in the process was designed to be a Reactor module with two standard Luer ports in the top for addition of materials and the application of vacuum or increased pressure for transfer of materials into subsequent cartridges. This initial Reactor module is connected to the second module via a $3 \mathrm{~mm}$ diameter S-Connector. Once the reaction mixture is dissolved it is transferred to a second module.

In this second module it is treated with acid, and $\mathbf{1}$ is isolated as a white solid. At this point, it is redissolved in DCM and treated with oxalyl chloride and catalytic dimethylformamide (DMF) to activate the carbonyl group by making the acyl 
a

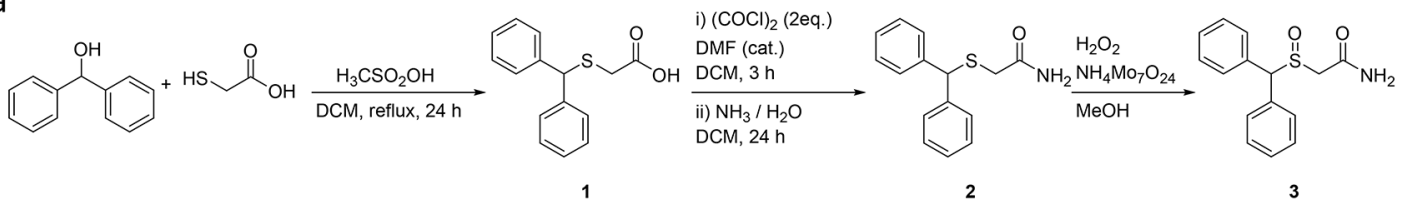

b
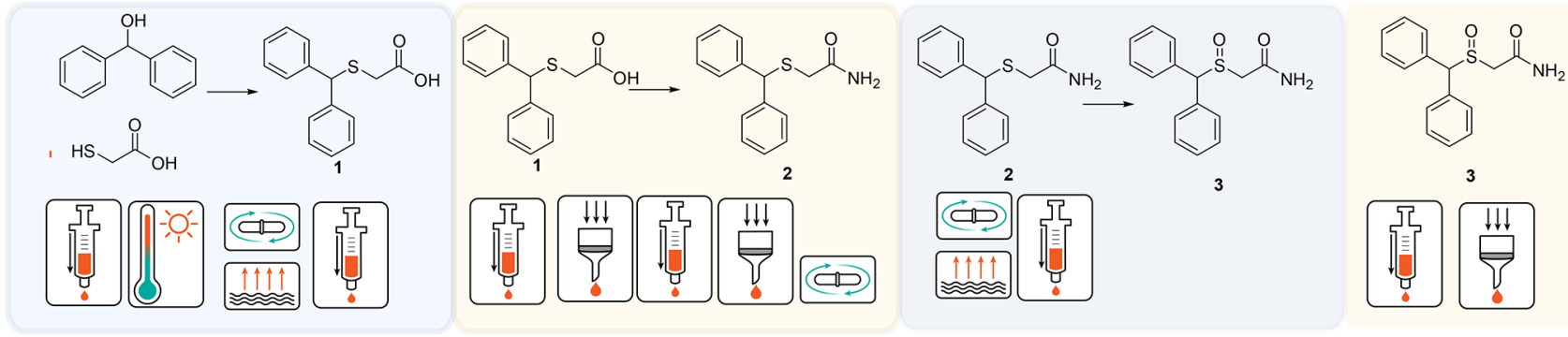

C

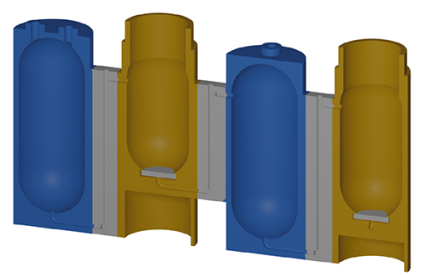

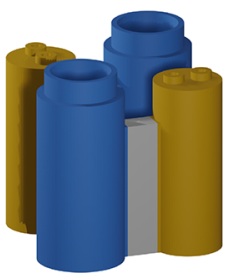

d

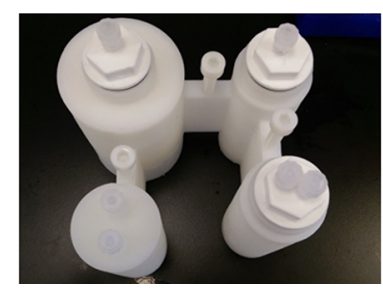

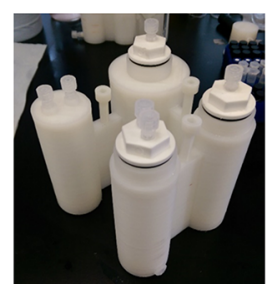

Figure 4. Schematic representation of the translation of the synthesis of Modafinil into a reactionware cartridge using ChemSCAD. (a) Synthetic route for Modafinil. (b) Reaction processes are split into groups, which can be assigned to specific reactor module types. (c) Monolithic synthesis cartridge generated from the combination of the identified reactor modules, depicted in either a linear (left) or print-optimized (right) format. (d) Final cartridges fabricated from the ChemSCAD designs.

chloride in situ. In the same cartridge aqueous ammonia is added with vigorous stirring, forming the amide (compound 2 ). This mixture is then filtered, and the filtrate, containing 2 , is retained for the next step. All of these procedures can therefore be carried out in a single Filter Reactor type of module, with the reaction mixture being transferred (leaving any precipitate in the Filter Reactor) into a third Reactor module for further processing. Once in this third module the resulting filtrate is concentrated to afford compound $\mathbf{2}$ as a pale yellow solid. Compound $\mathbf{2}$ is then dissolved in methanol and treated with a hydrogen peroxide along with catalytic ammonium heptamolybdate to carry out the final sulfoxidation. This process requires a Reactor module similar to the first. In order to isolate the final product this reaction mixture must then be transferred into a final, Filter Reactor module, where the desired material is precipitated from an aqueous solution and Modafinil (compound 3) is obtained in good purity (95\%) and yield (60\% overall yield). Further details of all syntheses carried out, along with detailed operation tables for the processes described, can be found in the Supporting Information.

The reactionware cartridge for the synthesis of Modafinil, therefore, consists of four modules: a Reactor module followed by a Filter Reactor, another Reactor, and the final Filter Reactor (see Figure 4). The first module consists of two standard Luer ports and a simple reactor bottom; it is connected to the second reactor through a $3 \mathrm{~mm}$ diameter cannula, which is equipped with a screw-valve. The second module features a threaded-cap top and a Filter Reactor bottom. The third Reactor module is the same as the first one, and the final Filter Reactor has the same top as the second Filter Reactor; the bottom differs in that it is the last reactor and requires a port for waste removal. The order and nature of each of the modules is defined entirely by the requirements of the synthetic procedure. When a similar design process is carried out for the synthesis of Lomustine, the cartridge consists of two Filter Reactor modules joined by an S-Connector that includes a drill pilot hole. The drill pilot hole aids the correct vertical alignment when drilling and tapping the printed monolith to allow screw-valve insertion. The screw-valve can be used to either allow or prevent the passage of liquid from one module to the next, or it can be completely opened and used as a port for waste disposal. The two Filter Reactors are fitted with two threaded caps, each of which has an auxiliary valve at the top. Finally, the last port is used for filtrate disposal, but it could also be used for vacuum drying. In the first step (compound 4) is readily obtained by precipitation after the reaction of cyclohexane amine with 1-chloro-2-isocyanatoethane by using $\mathrm{Et}_{2} \mathrm{O}$ as solvent, then filtration, a wash by $\mathrm{Et}_{2} \mathrm{O}$, and a drying in module 1 . Compound 4 was redissolved in $\mathrm{HCOOH}$ and transferred to module 2 by applying compressed air. Following this, the nitrosation reaction is carried out by adding sodium nitrate in portions. After filtration and a wash with water, compound $\mathbf{5}$ (Lomustine) is obtained in high purity (99\%) and good yield (68\%). The schematic of the monolith and details of the reaction protocol as an operations table can be found in the Supporting Information.

In addition to the batch synthesis of Lomustine, ChemSCAD was used to design two flow reactors for continuous-flow synthesis. The reactors consist of a total of three ports, two input ports for the addition of reagent solutions and an output port for reaction mixture and product streams. All ports are connected to syringes containing reagents or to one another using polytetrafluoroethylene (PTFE) tubing and polyether ether ketone (PEEK) fittings embedded into the polypropylene (PP) flow reactors. The synthesis was carried out at a uniform flow rate of $0.04 \mathrm{~mL} / \mathrm{min}$. Both flow reactors have a total reaction volume of $0.56 \mathrm{~mL}$. Initially, a stream of cyclohexylamine and isocyanatoethane are combined in flow reactor 1 to form the urea intermediate in situ. The intermediate continues to flow into one of the two ports of 
flow reactor 2, where it is combined with a solution of tertbutyl nitrite. The final stream is a combination of Lomustine partitioned in DCM, tetrahydrofuran (THF), and acetonitrile (ACN). The collected mixture is worked up using water and diethyl ether, then concentrated to afford a yield of $98 \%$ of Lomustine. Further details can be found in the Supporting Information. Many syntheses of Ribavirin have been published since it was first reported. ${ }^{26-28}$ Considering the commercially available goods and cheap price, $1,2,3,5$-tetra- $O$-acetyl- $\beta$-Dribofuranose and methyl 1,2,4-triazole-3-carboxylate were chosen as starting materials.

The reactionware cartridge for the synthesis of Ribavirin is composed of three modules, a Reactor module followed by two Filter Reactor modules. All modules were equipped with the same type of $1 \mathrm{in}$. threaded cap and auxiliary ports. All modules are equipped with screw valves, and the final module has a port for waste disposal. In the first step the starting materials were melted at high temperature $\left(110{ }^{\circ} \mathrm{C}\right)$, and the reaction was catalyzed by $p$-toluenesulfonic acid $(\mathrm{TsOH})$ without any solvent present. The high concentration of reactants within the mixture of this reaction can easily block the glass filter of the module. To avoid this issue, a no-filter design was chosen for module 1 for the first step of the reaction sequence. After the reaction was finished, the mixture was dissolved using ethanol at an elevated temperature. Following the dissolution, the hot mixture was transferred through the filter and siphon tube to module 2 and cooled. Compound 6 was crystallized and isolated by filtration, washing, and drying in module 2 . Compound 7 is formed by treating compound $\mathbf{6}$ with methanolic ammonia, which dissolves it, and with continuous vigorous stirring a precipitate crashes out of solution. The mixture is filtered, and the crude reaction mixture is further dissolved using $80 \% \mathrm{EtOH}$ at $95{ }^{\circ} \mathrm{C}$ and transferred to module 3 by applying compressed air. After crystallization, filtration, and drying, compound 7 (Ribavirin) is obtained in high purity (98\%) and good overall yield (43\%).

\section{CONCLUSIONS}

In summary, the three target compounds (Lomustine, Ribavarin, and Modafinil) were synthesized in comparable yields and purities in contrast to traditional glassware syntheses. Because of the clear, precisely laid out step-by-step actions (detailed in the Supporting Information), using reactionware largely reduces the expertise necessary for the successful synthesis of each compound. Moreover, by using the 3D-printed geometry of the reactionware to constrain the operation of the synthesis, we reduce the human decision making involved in the synthesis processes, making the sequence more reproducible. That means reactionware protocols are less complex and time-consuming than traditional syntheses.

In conclusion, these syntheses demonstrate the methodology of translating multi-step, target-oriented syntheses into digitally defined reactor systems that allow chemists to easily design and iterate reactionware synthesis systems. The software developed to achieve this, ChemSCAD, is designed to be used intuitively by chemists using only chemically relevant information such as volumes and synthetic operations to produce modular designs, which can then be fabricated using $3 \mathrm{D}$ printers, or any other suitable method.

\section{EXPERIMENTAL SECTION}

Full details of the ChemSCAD system can be found in the Supporting Information, including the fabrication of the reactionware, the process for the synthesis of the compounds, and full analytical data; no unexpected or unusually high safety hazards were encountered during the course of this work. The open-source repository for ChemSCAD can be found here: https://pypi.org/project/chemscad/

\section{ASSOCIATED CONTENT}

\section{Supporting Information}

The Supporting Information is available free of charge at https://pubs.acs.org/doi/10.1021/acscentsci.0c01354.

Overview of the software (use of OpenSCAD and ccad, installation instructions, software layout, and ChemSCAD database), traditional/reactionware synthesis of drug molecules (Lomustine, Ribavirin, and Modafinil), and the corresponding analytical data (HPLC spectra, ${ }^{1} \mathrm{H}$ NMR spectra, ${ }^{13} \mathrm{C}$ NMR spectra, and HRMS data) (PDF) $A$ video detailing the installation of ChemSCAD on Windows (MP4)

\section{AUTHOR INFORMATION}

\section{Corresponding Author}

Leroy Cronin - School of Chemistry, The University of Glasgow, Glasgow G12 8QQ, U.K.; (i) orcid.org/00000001-8035-5757; Email: lee.cronin@glasgow.ac.uk

\section{Authors}

Wenduan Hou - School of Chemistry, The University of Glasgow, Glasgow G12 8QQ, U.K.

Andrius Bubliauskas - School of Chemistry, The University of Glasgow, Glasgow G12 8QQ U.K.

Philip J. Kitson - School of Chemistry, The University of Glasgow, Glasgow G12 8QQ U.K.

Jean-Patrick Francoia - School of Chemistry, The University of Glasgow, Glasgow G12 8QQ U.K.

Henry Powell-Davies - School of Chemistry, The University of Glasgow, Glasgow G12 8QQ, U.K.; (1) orcid.org/00000002-4665-5745

Juan Manuel Parrilla Gutierrez - School of Chemistry, The University of Glasgow, Glasgow G12 8QQ U.K.

Przemyslaw Frei - School of Chemistry, The University of Glasgow, Glasgow G12 8QQ U.K.

J. Sebastián Manzano - School of Chemistry, The University of Glasgow, Glasgow G12 8QQ U.K.

Complete contact information is available at:

https://pubs.acs.org/10.1021/acscentsci.0c01354

\section{Author Contributions}

$\dagger$ (W.H., A.B., and P.J.K.) These three authors contributed equally to this work.

\section{Author Contributions}

W.H., A.B., and P.J.K. contributed equally to this work. L.C. invented the concept and devised the project and the digitization approach, with help from P.J.K.; W.H. and A.B. designed and carried out traditional and reactionware synthetic routes to the prepared compounds. P.J.K. and L.C. wrote the paper with help from W.H., A.B., H.P.D., and S.M.; J.P.F. initially developed the ChemSCAD software, and H.P.D. and J.M.P. provided further development. W.H., A.B., J.S.M., and P.F. contributed to the design of specific reactionware cartridges for the presented syntheses. The authors acknowledge the help of Dr. C.-G. Lin for help with some of the syntheses and Dr. J. S. Mathieson and Dr. D. Castro for analytical support. 


\section{Funding}

We gratefully acknowledge financial support from the EPSRC (Grant Nos. EP/L023652/1, EP/R020914/1, EP/S030603/1, $\mathrm{EP} / \mathrm{R} 01308 X / 1, \mathrm{EP} / \mathrm{S} 017046 / 1$, and EP/S019472/1), the ERC (Project No. 670467 SMART-POM), the EC (Project No. 766975 MADONNA), The John Templeton Foundation (Project Nos. 60625 and 61184), and DARPA (Project Nos. W911NF-182-0036, W911NF-17-1-0316, and HR001119S0003).

Notes

The authors declare no competing financial interest.

\section{REFERENCES}

(1) Rue, W.; Afzal, D.; Karanassios, V. An overview of $3 D$ printing in chemistry; SPIE, 2020; Vol. 11390.

(2) Capel, A. J.; Rimington, R. P.; Lewis, M. P.; Christie, S. D. R. 3D printing for chemical, pharmaceutical and biological applications. Nature Reviews Chemistry 2018, 2, 422-436.

(3) Ahn, B. Y.; et al. Omnidirectional Printing of Flexible, Stretchable, and Spanning Silver Microelectrodes. Science 2009, 323, $1590-1593$.

(4) Therriault, D.; White, S. R.; Lewis, J. A. Chaotic mixing in threedimensional microvascular networks fabricated by direct-write assembly. Nat. Mater. 2003, 2, 265-271.

(5) Hasegawa, T.; Nakashima, K.; Omatsu, F.; Ikuta, K. Multidirectional micro-switching valve chip with rotary mechanism. Sens. Actuators, A 2008, 143, 390-398.

(6) Ilievski, F.; Mazzeo, A. D.; Shepherd, R. F.; Chen, X.; Whitesides, G. M. Soft Robotics for Chemists. Angew. Chem. 2011, 123, 1930-1935.

(7) Symes, M. D.; et al. Integrated 3D-printed reactionware for chemical synthesis and analysis. Nat. Chem. 2012, 4, 349-354.

(8) Kitson, P. J.; Rosnes, M. H.; Sans, V.; Dragone, V.; Cronin, L. Configurable 3D-Printed millifluidic and microfluidic 'lab on a chip' reactionware devices. Lab Chip 2012, 12, 3267.

(9) Kitson, P. J.; et al. 3D printing of versatile reactionware for chemical synthesis. Nat. Protoc. 2016, 11, 920-936.

(10) Kitson, P. J.; Marshall, R. J.; Long, D.; Forgan, R. S.; Cronin, L. $3 \mathrm{D}$ printed high-throughput hydrothermal reactionware for discovery, optimization, and scale-up. Angew. Chem., Int. Ed. 2014, 53, 1272312728 .

(11) Lin, C. G.; et al. Digital Control of Multistep Hydrothermal Synthesis by Using 3D Printed Reactionware for the Synthesis of Metal-Organic Frameworks. Angew. Chem., Int. Ed. 2018, 57, 1671616720 .

(12) Kitson, P. J.; et al. Digitization of multistep organic synthesis in reactionware for on-demand pharmaceuticals. Science 2018, 359, 314-319.

(13) Zalesskiy, S. S.; Kitson, P. J.; Frei, P.; Bubliauskas, A.; Cronin, L. 3D designed and printed chemical generators for on demand reagent synthesis. Nat. Commun. 2019, 10, 5496.

(14) Kitson, P. J.; et al. Digitization of multistep organic synthesis in reactionware for on-demand pharmaceuticals. Science 2018, 359, 314-319.

(15) Zalesskiy, S. S.; Kitson, P. J.; Frei, P.; Bubliauskas, A.; Cronin, L. 3D designed and printed chemical generators for on demand reagent synthesis. Nat. Commun. 2019, 10, 5496.

(16) Patterson, G. Modeling and Scale-Up of Mixing- and Temperature-Sensitive Chemical Reactions. Ind. Eng. Chem. Res. 2005, 44, 5325-5341.

(17) Paeshuyse, J.; Dallmeier, K.; Neyts, J. Ribavirin for the treatment of chronic hepatitis $\mathrm{C}$ virus infection: a review of the proposed mechanisms of action. Curr. Opin. Virol. 2011, 1, 590-598.

(18) Murillo-Rodríguez, E.; Barciela Veras, A.; Barbosa Rocha, N.; Budde, H.; Machado, S. An Overview of the Clinical Uses, Pharmacology, and Safety of Modafinil. ACS Chem. Neurosci. 2018, 9, 151-158.

(19) Chakkath, T.; Lavergne, S.; Fan, T. M.; Bunick, D.; Dirikolu, L. Alkylation and Carbamylation Effects of Lomustine and Its Major
Metabolites and MGMT Expression in Canine Cells. Vet Sci. 2015, 2, $52-68$.

(20) Introduction - Unit Operations. Ind. Eng. Chem. 1954 46, 5760, .

(21) Dach, R.; Song, J. J.; Roschangar, F.; Samstag, W.; Senanayake, C. H. The Eight Criteria Defining a Good Chemical Manufacturing Process. Org. Process Res. Dev. 2012, 16, 1697-1706.

(22) Aktoudianakis, E.; Lin, R. J.; Dicks, A. P. Keeping Your Students Awake: Facile Microscale Synthesis of Modafinil, a Modern Anti-Narcoleptic Drug. J. Chem. Educ. 2006, 83, 1832.

(23) Jung, J.-C.; et al. Simple synthesis of modafinil derivatives and their anti-inflammatory activity. Molecules 2012, 17, 10446-10458.

(24) Singer, C. G. N.; Ceausu, A.; Kuvernab, A.; Aronhime, J. Organic Substrates Having Improved Weatherability and Mar Resistance. Canada Patent CA 2685310C (2012).

(25) Lari, A.; et al. Synthesis and psychobiological evaluation of modafinil analogs in mice. Daru, J. Pharm. Sci. 2013, 21, 67-67.

(26) Li, Y. S.; Zhang, J. J.; Mei, L. Q.; Tan, C. X. An Improved Procedure for the Preparation of Ribavirin. Org. Prep. Proced. Int. 2012, 44, 387-391.

(27) Liu, W. Y.; et al. Synthesis of novel ribavirin hydrazone derivatives and anti-proliferative activity against A549 lung cancer cells. Carbohydr. Res. 2009, 344, 1270-1275.

(28) Rivero, C. W.; De Benedetti, E. C.; Lozano, M. E.; Trelles, J. A. Bioproduction of ribavirin by green microbial biotransformation. Process Biochem. 2015, 50, 935-940. 